\title{
On algebraic automorphisms and their rational invariants
}

\author{
Philippe Bonnet \\ Mathematisches Institut, Universität Basel \\ Rheinsprung 21, 4051 Basel, Switzerland \\ e-mail: Philippe.bonnet@unibas.ch
}

\begin{abstract}
Let $X$ be an affine irreducible variety over an algebraically closed field $k$ of characteristic zero. Given an automorphism $\Phi$, we denote by $k(X)^{\Phi}$ its field of invariants, i.e the set of rational functions $f$ on $X$ such that $f \circ \Phi=f$. Let $n(\Phi)$ be the transcendence degree of $k(X)^{\Phi}$ over $k$. In this paper, we study the class of automorphisms $\Phi$ of $X$ for which $n(\Phi)=\operatorname{dim} X-1$. More precisely, we show that under some conditions on $X$, every such automorphism is of the form $\Phi=\varphi_{g}$, where $\varphi$ is an algebraic action of a linear algebraic group $G$ of dimension 1 on $X$, and where $g$ belongs to $G$. As an application, we determine the conjugacy classes of automorphisms of the plane for which $n(\Phi)=1$.
\end{abstract}

\section{Introduction}

Let $k$ be an algebraically closed field of characteristic zero. Let $X$ be an affine irreducible variety of dimension $n$ over $k$. We denote by $\mathcal{O}(X)$ its ring of regular functions, and by $k(X)$ its field of rational functions. Given an algebraic automorphism $\Phi$ of $X$, denote by $\Phi^{*}$ the field automorphism induced by $\Phi$ on $k(X)$, i.e. $\Phi^{*}(f)=f \circ \Phi$ for any $f \in k(X)$. An element $f$ of $k(X)$ is invariant for $\Phi$ (or simply invariant) if $\Phi^{*}(f)=f$. Invariant rational functions form a field denoted $k(X)^{\Phi}$, and we set:

$$
n(\Phi)=\operatorname{trdeg}_{k} k(X)^{\Phi}
$$

In this paper, we are going to study the class of automorphisms of $X$ for which $n(\Phi)=$ $n-1$. There are natural candidates for such automorphisms, such as exponentials of locally nilpotent derivations (see [M] or [Da]). More generally, one can construct such automorphisms by means of algebraic group actions as follows. Let $G$ be a linear algebraic group over $k$. An algebraic action of $G$ on $X$ is a regular map:

$$
\varphi: G \times X \longrightarrow X
$$


of affine varieties, such that $\varphi\left(g \cdot g^{\prime}, x\right)=\varphi\left(g, \varphi\left(g^{\prime}, x\right)\right)$ for any $\left(g, g^{\prime}, x\right)$ in $G \times G \times X$. Given an element $g$ of $G$, denote by $\varphi_{g}$ the map $x \mapsto \varphi(g, x)$. Then $\varphi_{g}$ clearly defines an automorphism of $X$. Let $k(X)^{G}$ be the field of invariants of $G$, i.e. the set of rational functions $f$ on $X$ such that $f \circ \varphi_{g}=f$ for any $g \in G$. If $G$ is an algebraic group of dimension 1, acting faithfully on $X$, and if $g$ is an element of $G$ of infinite order, then one can prove by Rosenlicht's Theorem (see [Ro]) that:

$$
n\left(\varphi_{g}\right)=\operatorname{trdeg}_{k} k(X)^{G}=n-1
$$

We are going to see that, under some mild conditions on $X$, there are no other automorphisms with $n(\Phi)=n-1$ than those constructed above. In what follows, denote by $\mathcal{O}(X)^{\nu}$ the normalization of $\mathcal{O}(X)$, and by $G(X)$ the group of invertible elements of $\mathcal{O}(X)^{\nu}$.

Theorem 1.1 Let $X$ be an affine irreducible variety of dimension $n$ over $k$, such that $\operatorname{char}(k)=0$ and $G(X)^{*}=k^{*}$. Let $\Phi$ be an algebraic automorphism of $X$ such that $n(\Phi)=n-1$. Then there exist an abelian linear algebraic group $G$ of dimension 1 , and an algebraic action $\varphi$ of $G$ on $X$ such that $\Phi=\varphi_{g}$ for some $g \in G$ of infinite order.

Note that the structure of $G$ is fairly simple. Since every connected linear algebraic group of dimension 1 is either isomorphic to $G_{a}(k)=(k,+)$ or $G_{m}(k)=\left(k^{*}, \times\right)$ (see [Hum], p. 131), there exists a finite abelian group $H$ such that $G$ is either equal to $H \times G_{a}(k)$ or $H \times G_{m}(k)$. Moreover, the assumption on the group $G(X)$ is essential. Indeed, consider the automorphism $\Phi$ of $k^{*} \times k$ given by $\Phi(x, y)=(x, x y)$. Obviously, its field of invariants is equal to $k(x)$. However, it is easy to check that $\Phi$ cannot have the form given in the conclusion of Theorem 1.1.

This theorem is analogous to a result given by Van den Essen and Peretz (see [V-P]). More precisely, they establish a criterion to decide if an automorphism $\Phi$ is the exponential of a locally nilpotent derivation, based on the invariants and on the form of $\Phi$. A similar result has been developed by Daigle (see [Da]).

We apply these results to the group of automorphisms of the plane. First, we obtain a classification of the automorphisms $\Phi$ of $k^{2}$ for which $n(\Phi)=1$. Second, we derive a criterion on automorphisms of $k^{2}$ to have no nonconstant rational invariants.

Corollary 1.2 Let $\Phi$ be an algebraic automorphism of $k^{2}$. If $n(\Phi)=1$, then $\Phi$ is conjugate to one of the following forms:

- $\Phi_{1}(x, y)=\left(a^{n} x, a^{m} b y\right)$, where $(n, m) \neq(0,0), a, b \in k$, b is a root of unity but a is not,

- $\Phi_{2}(x, y)=(a x, b y+P(x))$, where $P$ belongs to $k[t]-\{0\}, a, b \in k$ are roots of unity.

Corollary 1.3 Let $\Phi$ be an algebraic automorphism of $k^{2}$. Assume that $\Phi$ has a unique fixpoint $p$ and that $d \Phi_{p}$ is unipotent. Then $n(\Phi)=0$. 
We then apply Corollary 1.3 to an automorphism of $\mathbb{C}^{3}$ recently discovered by Pierre-Marie Poloni and Lucy Moser (see [M-P]).

We may wonder whether Theorem 1.1 still holds if the ground field $k$ is not algebraically closed or has positive characteristic. The answer is not known for the moment. In fact, two obstructions appear in the proof of Theorem 1.1 when $k$ is arbitrary. First, the group $G_{m}(k)$ needs to be divisible (see Lemma 4.2), which is not always the case if $k$ is not algebraically closed. Second, the proof uses the fact that every $G_{a}(k)$-action on $X$ can be reconstructed from a locally nilpotent derivation on $\mathcal{O}(X)$ (see subsection 4.1), which is no longer true if $k$ has positive characteristic. This phenomenom is due to the existence of differents forms for the affine line (see $[\mathrm{Ru}])$. Note that, in case Theorem 1.1 holds and $k$ is not algebraically closed, the algebraic group $G$ needs not be isomorphic to $H \times G_{a}(k)$ or $H \times G_{m}(k)$, where $H$ is finite. Indeed consider the unit circle $X$ in the plane $\mathbb{R}^{2}$, given by the equation $x^{2}+y^{2}=1$. Let $\Phi$ be a rotation in $\mathbb{R}^{2}$ with center at the origin and angle $\theta \notin 2 \pi \mathbb{Q}$. Then $\Phi$ defines an algebraic automorphism of $X$ with $n(\Phi)=0$, and the subgroup spanned by $\Phi$ is dense in $S_{2}(\mathbb{R})$. But $S O_{2}(\mathbb{R})$ is not isomorphic to either $G_{a}(\mathbb{R})$ or $G_{m}(\mathbb{R})$, even though it is a connected linear algebraic group of dimension 1 .

We may also wonder what happens to the automorphisms $\Phi$ of $X$ for which $n(\Phi)=$ $\operatorname{dim} X-2$. More precisely, does there exist an action $\varphi$ of a linear algebraic group $G$ on $X$, of dimension 2, such that $\Phi=\varphi_{g}$ for a given $g \in G$ ? The answer is no. Indeed consider the automorphism $\Phi$ of $k^{2}$ given by $\Phi=f \circ g$, where $f(x, y)=\left(x+y^{2}, y\right)$ and $g(x, y)=\left(x, y+x^{2}\right)$. Let $d(n)$ denote the maximum of the homogeneous degrees of the coordinate functions of the iterate $\Phi^{n}$. If there existed an action $\varphi$ of a linear algebraic group $G$ such that $\Phi=\varphi_{g}$, then the function $d$ would be bounded, which is impossible since $d(n)=4^{n}$. A similar argument on the length of the iterates also yields the result. But if we restrict to some specific varieties $X$, for instance $X=k^{3}$, one may ask the following question: If $n(\Phi)=1$, is $\Phi$ birationally conjugate to an automorphism that leaves the first coordinate of $k^{3}$ invariant? The answer is still unknown.

\section{Reduction to an affine curve $\mathcal{C}$}

Let $X$ be an affine irreducible variety of dimension $n$ over $k$. Let $\Phi$ be an algebraic automorphism of $X$ such that $n(\Phi)=n-1$. In this section, we are going to construct an irreducible affine curve on which $\Phi$ acts naturally. This will allow us to use some well-known results on automorphisms of curves. We set:

$$
K=\left\{f \in k(X) \mid \exists m>0, f \circ \Phi^{m}=f \circ \Phi \circ \ldots \circ \Phi=f\right\}
$$

It is straightforward that $K$ is a subfield of $k(X)$ containing both $k$ and $k(X)^{\Phi}$. We begin with some properties of this field.

Lemma $2.1 K$ has transcendence degree $(n-1)$ over $k$, and is algebraically closed in $k(X)$. In particular, the automorphism $\Phi$ of $X$ has infinite order. 
Proof: First we show that $K$ has transcendence degree $(n-1)$ over $k$. Since $K$ contains the field $k(X)^{\Phi}$, whose transcendence degree is $(n-1)$, we only need to show that the extension $K / k(X)^{\Phi}$ is algebraic, or in other words that every element of $K$ is algebraic over $k(X)^{\Phi}$. Let $f$ be any element of $K$. By definition, there exists an integer $m>0$ such that $f \circ \Phi^{m}=f$. Let $P(t)$ be the polynomial of $k(X)[t]$ defined as:

$$
P(t)=\prod_{i=0}^{m-1}\left(t-f \circ \Phi^{i}\right)
$$

By construction, the coefficients of this polynomial are all invariant for $\Phi$, and $P(t)$ belongs to $k(X)^{\Phi}[t]$. Moreover $P(f)=0, f$ is algebraic over $k(X)^{\Phi}$ and the first assertion follows.

Second we show that $K$ is algebraically closed in $k(X)$. Let $f$ be an element of $k(X)$ that is algebraic over $K$. We need to prove that $f$ belongs to $K$. By the first assertion of the lemma, $f$ is algebraic over $k(X)^{\Phi}$. Let $P(t)=a_{0}+a_{1} t+\ldots+a_{p} t^{p}$ be a nonzero minimal polynomial of $f$ over $k(X)^{\Phi}$. Since $P(f)=0$ and all $a_{i}$ are invariant, we have $P(f \circ \Phi)=P(f) \circ \Phi=0$. In particular, all elements of the form $f \circ \Phi^{i}$, with $i \in \mathbb{N}$, are roots of $P$. Since $P$ has finitely many roots, there exist two distinct integers $m^{\prime}<m^{\prime \prime}$ such that $f \circ \Phi^{m^{\prime}}=f \circ \Phi^{m^{\prime \prime}}$. In particular, $f \circ \Phi^{m^{\prime \prime}-m^{\prime}}=f$ and $f$ belongs to $K$.

Now if $\Phi$ were an automorphism of finite order, then $K$ would be equal to $k(X)$. But this is impossible since $K$ and $k(X)$ have different transcendence degrees.

Lemma 2.2 There exists an integer $m>0$ such that $K=k(X)^{\Phi^{m}}$.

Proof: By definition, $k(X)$ is a field of finite type over $k$. Since $K$ is contained in $k(X)$, $K$ has also finite type over $k$. Let $f_{1}, \ldots, f_{r}$ be some elements of $k(X)$ such that $K=$ $k\left(f_{1}, \ldots, f_{r}\right)$. Let $m_{1}, \ldots, m_{r}$ be some positive integers such that $f_{i} \circ \Phi^{m_{i}}=f_{i}$, and set $m=m_{1} \ldots m_{r}$. By construction, all $f_{i}$ are invariant for $\Phi^{m}$. In particular, $K$ is invariant for $\Phi^{m}$ and $K \subseteq k(X)^{\Phi^{m}}$. Since $k(X)^{\Phi^{m}} \subseteq K$, the result follows.

Let $L$ be the algebraic closure of $k(X)$, and let $A$ be the $K$-subalgebra of $L$ spanned by $\mathcal{O}(X)$. By construction, $A$ is an integral $K$-algebra of finite type of dimension 1 . Let $m$ be an integer satisfying the conditions of lemma 2.2. The automorphism $\Psi^{*}=\left(\Phi^{m}\right)^{*}$ of $\mathcal{O}(X)$ stabilizes $A$, hence it defines a $K$-automorphism of $A$, of infinite order (see lemma 2.1). Let $B$ be the integral closure of $A$. Then $B$ is also an integral $K$-algebra of finite type, of dimension 1 , and the $K$-automorphism $\Psi^{*}$ extends uniquely to $B$. If $\bar{K}$ stands for the algebraic closure of $K$, we set:

$$
C=B \otimes_{K} \bar{K}
$$

By construction, $\mathcal{C}=\operatorname{Spec}(C)$ is an affine curve over the algebraically closed field $\bar{K}$. Moreover the automorphism $\Psi^{*}$ acts on $C$ via the operation:

$$
\Psi^{*}: C \longrightarrow C, \quad x \otimes y \longmapsto \Psi^{*}(x) \otimes y
$$


This makes sense since $\Psi^{*}$ fixes the field $K$. Therefore $\Psi^{*}$ induces an algebraic automorphism of the curve $\mathcal{C}$. Since $K$ is algebraically closed in $k(X)$ by lemma 2.1, $C$ is integral (see [Z-S], Chap. VII, $\S 11$, Theorem 38). But by construction, $B$ and $\bar{K}$ are normal rings. Since $C$ is a domain and $\operatorname{char}(K)=0, C$ is also integrally closed by a result of Bourbaki (see [Bou], p. 29). So $C$ is a normal domain and $\mathcal{C}$ is a smooth irreducible curve.

Lemma 2.3 Let $C$ be the $\bar{K}$-algebra constructed above. Then either $C=\bar{K}[t]$ or $C=$ $\bar{K}[t, 1 / t]$.

Proof: By lemma 2.1, the automorphism $\Phi$ of $X$ has infinite order. Since the fraction field of $B$ is equal to $k(X), \Psi^{*}$ has infinite order on $B$. But $B \otimes 1 \subset C$, so $\Psi^{*}$ has infinite order on $C$. In particular, $\Psi$ acts like an automorphism of infinite order on $\mathcal{C}$. Since $\mathcal{C}$ is affine, it has genus zero (see [Ro2]). Since $\bar{K}$ is algebraically closed, the curve $\mathcal{C}$ is rational (see [Che], p. 23 ). Since $\mathcal{C}$ is smooth, it is isomorphic to $\mathbb{P}^{1}(\bar{K})-E$, where $E$ is a finite set. Moreover, $\Psi$ acts like an automorphism of $\mathbb{P}^{1}(\bar{K})$ that stabilizes $\mathbb{P}^{1}(\bar{K})-E$. Up to replacing $\Psi$ by one of its iterates, we may assume that $\Psi$ fixes every point of $E$. But an automorphism of $\mathbb{P}^{1}(\bar{K})$ that fixes at least three points is the identity, which is impossible. Therefore $E$ consists of at most two points, and $\mathcal{C}$ is either isomorphic to $\bar{K}$ or to $\bar{K}^{*}$. In particular, either $C=\bar{K}[t]$ or $C=\bar{K}[t, 1 / t]$.

\section{Normal forms for the automorphism $\Psi$}

Let $C$ and $\Psi^{*}$ be the $\bar{K}$-algebra and the $\bar{K}$-automorphism constructed in the previous section. In this section, we are going to give normal forms for the couple $\left(C, \Psi^{*}\right)$, in case the group $G(X)$ is trivial, i.e. $G(X)=k^{*}$. We begin with a few lemmas.

Lemma 3.1 Let $X$ be an irreducible affine variety over $k$. Let $\Psi$ be an automorphism of $X$. Let $\alpha, f$ be some elements of $k(X)^{*}$ such that $\left(\Psi^{*}\right)^{n}(f)=\alpha^{n} f$ for any $n \in \mathbb{Z}$. Then $\alpha$ belongs to $G(X)$.

Proof: Given an element $h$ of $k(X)^{*}$ and a prime divisor $D$ on the normalization $X^{\nu}$, we consider $h$ as a rational function on $X^{\nu}$, and denote by $\operatorname{ord}_{D}(h)$ the multiplicity of $h$ along $D$. This makes sense since the variety $X^{\nu}$ is normal. Fix any prime divisor $D$ on $X$. Since $\left(\Psi^{*}\right)^{n}(f)=\alpha^{n} f$ for any $n \in \mathbb{Z}$, we obtain:

$$
\operatorname{ord}_{D}\left(\left(\Psi^{*}\right)^{n}(f)\right)=n \operatorname{ord}_{D}(\alpha)+\operatorname{ord}_{D}(f)
$$

Since $\Psi$ is an algebraic automorphism of $X$, it extends uniquely to an algebraic automorphism of $X^{\nu}$, which is still denoted $\Psi$. Moreover, this extension maps every prime divisor to another prime divisor, does not change the multiplicity and maps distinct prime divisors into distinct ones. If $\operatorname{div}(f)=\sum_{i} n_{i} D_{i}$, where all $D_{i}$ are prime, then we have:

$$
\operatorname{div}\left(\left(\Psi^{*}\right)^{n}(f)\right)=\sum_{i} n_{i}\left(\Psi^{*}\right)^{n}\left(D_{i}\right)
$$


where all $\left(\Psi^{*}\right)^{n}\left(D_{i}\right)$ are prime and distinct. So the multiplicity of $\left(\Psi^{*}\right)^{n}(f)$ along $D$ is equal to zero if $D$ is none of the $\left(\Psi^{*}\right)^{n}\left(D_{i}\right)$, and equal to $n_{i}$ if $D=\left(\Psi^{*}\right)^{n}\left(D_{i}\right)$. In all cases, if $R=\max \left\{\left|n_{i}\right|\right\}$, then we find that $\left|\operatorname{ord}_{D}\left(\left(\Psi^{*}\right)^{n}(f)\right)\right| \leq R$ and $\left|\operatorname{ord}_{D}(f)\right| \leq R$, and this implies for any integer $n$ :

$$
\left|n \operatorname{ord}_{D}(\alpha)\right| \leq 2 R
$$

In particular we find $\operatorname{ord}_{D}(\alpha)=0$. Since this holds for any prime divisor $D$, the support of $\operatorname{div}(\alpha)$ in $X^{\nu}$ is empty and $\operatorname{div}(\alpha)=0$. Since $X^{\nu}$ is normal, $\alpha$ is an invertible element of $\mathcal{O}(X)^{\nu}$, hence it belongs to $G(X)$.

Lemma 3.2 Let $K$ be a field of characteristic zero and $\bar{K}$ its algebraic closure. Let $C$ be either equal to $\bar{K}[t]$ or to $\bar{K}[t, 1 / t]$. Let $\Psi^{*}$ be a $\bar{K}$-automorphism of $C$ such that $\Psi^{*}(t)=$ at, where a belongs to $\bar{K}$. Let $\sigma_{1}$ be a $K$-automorphism of $C$, commuting with $\Psi^{*}$, such that $\sigma_{1}(\bar{K})=\bar{K}$. Then $\sigma_{1}(a)$ is either equal to a or to $1 / a$.

Proof: We distinguish two cases depending on the ring $C$. First assume that $C=\bar{K}[t]$. Since $\sigma_{1}$ is a $K$-automorphism of $C$ that maps $\bar{K}$ to itself, we have $\bar{K}[t]=\bar{K}\left[\sigma_{1}(t)\right]$. In particular $\sigma_{1}(t)=\lambda t+\mu$, where $\lambda, \mu$ belong to $\bar{K}$ and $\lambda \neq 0$. Since $\Psi^{*}$ and $\sigma_{1}$ commute, we obtain:

$$
\Psi^{*} \circ \sigma_{1}(t)=\lambda a t+\mu=\sigma_{1} \circ \Psi^{*}(t)=\sigma_{1}(a)(\lambda t+\mu)
$$

In particular, we have $\sigma_{1}(a)=a$ and the lemma follows in this case. Second assume that $C=\bar{K}[t, 1 / t]$. Since $\sigma_{1}$ is a $K$-automorphism of $C$, we find:

$$
\sigma_{1}(t) \sigma_{1}(1 / t)=\sigma_{1}(t .1 / t)=\sigma_{1}(1)=1
$$

Therefore $\sigma_{1}(t)$ is an invertible element of $C$, and has the form $\sigma_{1}(t)=a_{1} t^{n_{1}}$, where $a_{1} \in \bar{K}^{*}$ and $n_{1}$ is an integer. Since $\sigma_{1}$ is a $K$-automorphism of $C$ that maps $\bar{K}$ to $\bar{K}$, we have $\bar{K}[t, 1 / t]=\bar{K}\left[\sigma_{1}(t), 1 / \sigma_{1}(t)\right]$. In particular $\left|n_{1}\right|=1$ and either $\sigma_{1}(t)=a_{1} t$ or $\sigma_{1}(t)=a_{1} / t$. If $\sigma_{1}(t)=a_{1} t$, the relation $\Psi^{*} \circ \sigma_{1}(t)=\sigma_{1} \circ \Psi^{*}(t)$ yields $\sigma(a)=a$. If $\sigma_{1}(t)=a_{1} / t$, then the same relation yields $\sigma(a)=1 / a$.

Lemma 3.3 Let $X$ be an irreducible affine variety of dimension $n$ over $k$, such that $G(X)=k^{*}$. Let $\Phi$ be an automorphism of $X$ such that $n(\Phi)=(n-1)$. Let $\Psi^{*}$ be the automorphism of $C$ constructed in the previous section. If either $C=\bar{K}[t]$ or $C=\bar{K}[t, 1 / t]$, and if $\Psi^{*}(t)=$ at, then a belongs to $k^{*}$.

Proof: We are going to prove by contradiction that $a$ belongs to $k^{*}$. So assume that $a \notin k^{*}$. Let $\sigma$ be any element of $\operatorname{Gal}(\bar{K} / K)$, and denote by $\sigma_{1}$ the $K$-automorphism of $C$ defined as follows:

$$
\forall(x, y) \in B \times \bar{K}, \sigma_{1}(x \otimes y)=x \otimes \sigma_{1}(y)
$$


Since $\Psi^{*} \circ \sigma_{1}(x \otimes y)=\Psi^{*}(x) \otimes \sigma_{1}(y)=\sigma_{1} \circ \Psi^{*}(x \otimes y)$ for any element $x \otimes y$ of $B \otimes_{K} \bar{K}, \Psi^{*}$ and $\sigma_{1}$ commute. Moreover if we identify $\bar{K}$ with $1 \otimes \bar{K}$, then $\sigma_{1}(\bar{K})=\bar{K}$ by construction. By lemma 3.2, we obtain:

$$
\forall \sigma \in \operatorname{Gal}(\bar{K} / K), \quad \sigma(a)=a \quad \text { or } \quad \sigma(a)=\frac{1}{a}
$$

In particular, the element $\left(a^{i}+a^{-i}\right)$ is invariant under the action of $\operatorname{Gal}(\bar{K} / K)$ for any $i$, and so it belongs to $K$ because $\operatorname{char}(K)=0$. Now let $f$ be an element of $B-K$. Since $f$ belongs to $C$, we can express $f$ as follows:

$$
f=\sum_{i=r}^{s} f_{i} t^{i}
$$

Choose an $f \in B-K$ such that the difference $(s-r)$ is minimal. We claim that $(s-r)=0$, i.e. $f=f_{s} t^{s}$. Indeed, assume that $s>r$. Since $f$ is an element of $B$, the following expressions:

$$
\begin{aligned}
& \Psi^{*}(f)+\left(\Psi^{*}\right)^{-1}(f)-\left(a^{s}+a^{-s}\right) f=\sum_{i=r}^{s-1} f_{i}\left(a^{i}+a^{-i}-a^{s}-a^{-s}\right) t^{i} \\
& \Psi^{*}(f)+\left(\Psi^{*}\right)^{-1}(f)-\left(a^{r}+a^{-r}\right) f=\sum_{i=r+1}^{s} f_{i}\left(a^{i}+a^{-i}-a^{r}-a^{-r}\right) t^{i}
\end{aligned}
$$

also belong to $B$. By minimality of $(s-r)$, these expressions belong to $K$. In other words, $f_{i}\left(a^{i}+a^{-i}-a^{s}-a^{-s}\right)=0$ (resp. $\left.f_{i}\left(a^{i}+a^{-i}-a^{r}-a^{-r}\right)=0\right)$ for any $i \neq 0, s$ (resp. for any $i \neq 0, r)$. Since $k$ is algebraically closed and $a \notin k^{*}$ by assumption, $\left(a^{i}+a^{-i}-a^{s}-a^{-s}\right)$ (resp. $\left(a^{i}+a^{-i}-a^{r}-a^{-r}\right)$ ) is nonzero for any $i \neq s$ (resp. for any $i \neq r$ ). Therefore $f_{i}=0$ for any $i \neq 0$, and $f$ belongs to $K$, a contradiction. Therefore $s=r$ and $f=f_{s} t^{s}$. Since $f$ belongs to $B$, it also belongs to $k(X)$. Since $\Psi$ is an automorphism of $X$, the element $a^{s}=\Psi^{*}(f) / f$ belongs to $k(X)$. Moreover $\left(\Psi^{*}\right)^{n}(f)=a^{n s} f$ for any $n \in \mathbb{Z}$. By lemma 3.1, $a^{s}$ belongs to $G(X)=k^{*}$. Since $k$ is algebraically closed, $a$ belongs to $k^{*}$, hence a contradiction, and the result follows.

Proposition 3.4 Let $X$ be an irreducible affine variety of dimension $n$ over $k$, such that $G(X)=k^{*}$. Let $\Phi$ be an automorphism of $X$ such that $n(\Phi)=(n-1)$. Let $C$ and $\Psi^{*}$ be the $\bar{K}$-algebra and the $\bar{K}$-automorphism constructed in the previous section. Then up to conjugation, one of the following three cases occurs:

- $C=\bar{K}[t]$ and $\Psi^{*}(t)=t+1$,

- $C=\bar{K}[t]$ and $\Psi^{*}(t)=$ at, where $a \in k^{*}$ is not a root of unity,

- $C=\bar{K}[t, 1 / t]$ and $\Psi^{*}(t)=$ at, where $a \in k^{*}$ is not a root of unity. 
Proof: By lemma 2.3, we know that either $C=\bar{K}[t]$ or $C=\bar{K}[t, 1 / t]$. We are going to study both cases.

First case: $C=\bar{K}[t]$.

The automorphism $\Psi^{*}$ maps $t$ to $a t+b$, where $a \in \bar{K}^{*}$ and $b \in \bar{K}$. If $a=1$, then $b \neq 0$ and up to replacing $t$ with $t / b$, we may assume that $\Psi^{*}(t)=t+1$. If $a \neq 1$, then up to replacing $t$ with $t-c$ for a suitable $c$, we may assume that $\Psi^{*}(t)=a t$. But then lemma 3.3 implies that $a$ belongs to $k^{*}$. Since $\Psi^{*}$ has infinite order, $a$ cannot be a root of unity.

Second case: $C=\bar{K}[t, 1 / t]$.

Since $\Psi^{*}(t) \Psi^{*}(1 / t)=\Psi^{*}(1)=1, \Psi^{*}(t)$ is an invertible element of $C$. So $\Psi^{*}(t)=a t^{n}$, where $a \in \bar{K}^{*}$ and $n \neq 0$. Since $\Psi^{*}$ is an automorphism, $n$ is either equal to 1 or to -1 . But if $n$ were equal to -1 , then a simple computation shows that $\left(\Psi^{*}\right)^{2}$ would be the identity, which is impossible. So $\Psi^{*}(t)=a t$, where $a \in \bar{K}^{*}$. By lemma 3.3, a belongs to $k^{*}$. As before, a cannot be a root of unity.

\section{Proof of the main theorem}

In this section, we are going to establish Theorem 1.1. We will split its proof in two steps depending on the form of the automorphism $\Psi^{*}$ given in Proposition 3.4 . But before, we begin with a few lemmas.

Lemma 4.1 Let $\Phi$ be an automorphism of an affine irreducible variety $X$. Let $G$ be a linear algebraic group and $\psi$ be an algebraic $G$-action on $X$. Let $h$ be an element of $G$ such that the group $\langle h\rangle$ spanned by $h$ is Zariski dense in $G$. If $\Phi$ and $\psi_{h}$ commute, then $\Phi$ and $\psi_{g}$ commute for any $g$ in $G$.

Proof: It suffices to check that $\Phi^{*}$ and $\psi_{g}^{*}$ commute for any $g \in G$. For any $k$-algebra automorphisms $\alpha, \beta$ of $\mathcal{O}(X)$, denote by $[\alpha, \beta]$ their commutator, i.e. $[\alpha, \beta]=\alpha \circ \beta \circ \alpha^{-1} \circ$ $\beta^{-1}$. For any $f \in \mathcal{O}(X)$, set:

$$
\lambda(g, f)(x)=\left[\Phi^{*}, \psi_{g}^{*}\right](f)(x)-f(x)
$$

Since $G$ is a linear algebraic group acting algebraically on the affine variety $X, \lambda(g, f)(x)$ is a regular function on $G \times X$. Since $\Phi^{*}$ and $\psi_{h}^{*}$ commute, the automorphisms $\Phi^{*}$ and $\psi_{h^{n}}^{*}$ commute for any integer $n$. So the regular function $\lambda(g, f)(x)$ vanishes on $\langle h\rangle \times X$. Since $\langle h\rangle$ is dense in $G$ by assumption, $\langle h\rangle \times X$ is dense in $G \times X$ and $\lambda(g, f)(x)$ vanishes identically on $G \times X$. In particular, $\left[\Phi^{*}, \psi_{g}^{*}\right](f)=f$ for any $g \in G$. Since this holds for any element $f$ of $\mathcal{O}(X)$, the bracket $\left[\Phi^{*}, \psi_{g}^{*}\right]$ coincides with the identity on $\mathcal{O}(X)$ for any $g \in G$, and the result follows. 
Lemma 4.2 Let $\Phi$ be an automorphism of an affine irreducible variety $X$. Let $G$ be a linear algebraic group and $\psi$ be an algebraic $G$-action on $X$. Let $h$ be an element of $G$ such that the group $\langle h\rangle$ spanned by $h$ is Zariski dense in $G$. Assume there exists a nonzero integer $r$ such that $\Phi^{r}=\psi_{h}$, and that $G$ is divisible. Then there exists an algebraic action $\varphi$ of $G^{\prime}=\mathbb{Z} / r \mathbb{Z} \times G$ such that $\Phi=\varphi_{g^{\prime}}$ for some $g^{\prime}$ in $G^{\prime}$.

Proof: Fix an element $b$ in $G$ such that $b^{r}=h$, and set $\Delta=\Phi \circ \psi_{b^{-1}}$. This is possible since $G$ is divisible. By construction, $\Delta$ is an automorphism of $X$. Since $\Phi^{r}=\psi_{h}, \Phi$ and $\psi_{h}$ commute. By lemma 4.1, $\Phi$ and $\psi_{g}$ commute for any $g \in G$. In particular, we have:

$$
\Delta^{r}=\left(\Phi^{r}\right) \circ \psi_{b^{-r}}=\left(\Phi^{r}\right) \circ \psi_{h^{-1}}=\mathrm{Id}
$$

So $\Delta$ is finite, $\Phi=\Delta \circ \psi_{b}$ and $\Delta$ commutes with $\psi_{g}$ for any $g \in G$. The group $G^{\prime}$ then acts on $X$ via the map $\varphi$ defined by:

$$
\varphi_{(i, g)}(x)=\Delta^{i} \circ \psi_{g}(x)
$$

Moreover we have $\Phi=\varphi_{g^{\prime}}$ for $g^{\prime}=(1, b)$.

The proof of Theorem 1.1 will then go as follows. In the following subsections, we are going to exhibit an algebraic action $\psi$ of $G_{a}(k)$ (resp. $G_{m}(k)$ ) on $X$, such that $\Psi=\Phi^{m}=\psi_{h}$ for some $h$. In both cases, the group $G$ we will consider will be linear algebraic of dimension 1, and divisible. Moreover the element $h$ will span a Zariski dense set because $h \neq 0$ (resp. $h$ is not a root of unity). With these conditions, Theorem 1.1 will become a direct application of Lemma 4.2,

\subsection{The case $\Psi^{*}(t)=t+1$}

Assume that $C=\bar{K}[t]$ and $\Psi^{*}(t)=t+1$. We are going to construct a nontrivial algebraic $G_{a}(k)$-action $\psi$ on $X$ such that $\Psi=\psi_{1}$. Since $\mathcal{O}(X) \subset C$, every element $f$ of $\mathcal{O}(X)$ can be written as $f=P(t)$, where $P$ belongs to $\bar{K}[t]$. We set $r=\operatorname{deg}_{t} P(t)$. Since $\Psi^{*}$ stabilizes $\mathcal{O}(X)$, the expression:

$$
\left(\Psi^{i}\right)^{*}(f)=P(t+i)=\sum_{j=0}^{r} P^{(j)}(t) \frac{i^{j}}{j !}
$$

belongs to $\mathcal{O}(X)$ for any integer $i$. Since the matrix $M=\left(i^{j} / j !\right)_{0 \leq i, j \leq r}$ is invertible in $\mathcal{M}_{r+1}(\mathbb{Q})$, the polynomial $P^{(j)}(t)$ belongs to $\mathcal{O}(X)$ for any $j \leq r$. So the $\bar{K}$-derivation $D=\partial / \partial t$ on $C$ stabilizes the $k$-algebra $\mathcal{O}(X)$. Since $D^{r+1}(f)=0$, the operator $D$, considered as a $k$-derivation on $\mathcal{O}(X)$, is locally nilpotent (see [Van]). Therefore the exponential map:

$$
\exp u D: \mathcal{O}(X) \longrightarrow \mathcal{O}(X)[u], \quad f \longmapsto \sum_{j \geq 0} D^{j}(f) \frac{u^{j}}{j !}
$$


is a well-defined $k$-algebra morphism. But exp $u D$ defines also a $K$-algebra morphism from $C$ to $C[u]$. Since $\exp u D(t)=t+u, \exp D$ coincides with $\Psi^{*}$ on $C$. Since $C$ contains the ring $\mathcal{O}(X)$, we have $\exp D=\Psi^{*}$ on $\mathcal{O}(X)$. So the exponential map induces an algebraic $G_{a}(k)$-action $\psi$ on $X$ such that $\Psi=\psi_{1}$ (see [Van]).

\subsection{The case $\Psi^{*}(t)=a t$}

Assume that $\Psi^{*}(t)=a t$ and $a$ is not a root of unity. We are going to construct a nontrivial algebraic $G_{m}(k)$-action $\psi$ on $X$ such that $\Psi=\psi_{a}$. First note that either $C=\bar{K}[t]$ or $C=\bar{K}[t, 1 / t]$. Let $f$ be any element of $\mathcal{O}(X)$. Since $\mathcal{O}(X) \subset C$, we can write $f$ as:

$$
f=P(t)=\sum_{i=r}^{s} f_{i} t^{i}
$$

where the $f_{i} t^{i}$ belong a priori to $C$. Since $\Psi^{*}$ stabilizes $\mathcal{O}(X)$, the expression:

$$
\left(\Psi^{j}\right)^{*}(f)=P\left(a^{j} t\right)=\sum_{i=r}^{s} a^{j i} f_{i} t^{i}
$$

belongs to $\mathcal{O}(X)$ for any integer $j$. Since $a$ belongs to $k^{*}$ and is not a root of unity, the Vandermonde matrix $M=\left(a^{i j}\right)_{0 \leq i, j \leq s-r}$ is invertible in $\mathcal{M}_{s-r+1}(k)$. So the elements $f_{i} t^{i}$ all belong to $\mathcal{O}(X)$ for any integer $i$. Consider the map:

$$
\psi^{*}: \mathcal{O}(X) \longrightarrow \mathcal{O}(X)[v, 1 / v], \quad f \longmapsto \sum_{i=r}^{s} f_{i} t^{i} v^{i}
$$

Then $\psi^{*}$ is a well-defined $k$-algebra morphism, which induces a regular map $\psi$ from $k^{*} \times X$ to $X$. Moreover we have $\psi_{v} \circ \psi_{v^{\prime}}=\psi_{v v^{\prime}}$ on $X$ for any $v, v^{\prime} \in k^{*}$. So $\psi$ defines an algebraic $G_{m}(k)$-action on $X$ such that $\Psi=\psi_{a}$.

\section{Proof of Corollary 1.2}

Let $\Phi$ be an automorphism of the affine plane $k^{2}$, such that $n(\Phi)=1$. By Theorem 1.1, there exists an algebraic action $\varphi$ of an abelian linear algebraic group $G$ of dimension 1 such that $\Phi=\varphi_{g}$. We will distinguish the cases $G=\mathbb{Z} / r \mathbb{Z} \times G_{m}(k)$ and $G=\mathbb{Z} / r \mathbb{Z} \times G_{a}(k)$.

First case: $G=\mathbb{Z} / r \mathbb{Z} \times G_{m}(k)$.

Then $G$ is linearly reductive and $\varphi$ is conjugate to a representation in $G l_{2}(k)$ (see [Ka] or $[\mathrm{Kr}])$. Since $G$ consists solely of semisimple elements, $\varphi$ is even diagonalizable. In particular, there exists a system $(x, y)$ of polynomial coordinates, some integers $n, m$ and some $r$-roots of unity $a, b$ such that:

$$
\varphi_{(i, u)}(x, y)=\left(a^{i} u^{n} x, b^{i} u^{m} y\right)
$$


Note that, since the action is faithful, the couple $(n, m)$ is distinct from $(0,0)$. Since $k$ is algebraically closed, we can even reduce $\Phi=\varphi_{g}$ to the first form given in Corollary 1.2 ,

Second case: $G=\mathbb{Z} / r \mathbb{Z} \times G_{a}(k)$.

Let $\psi$ and $\Delta$ be respectively the $G_{a}(k)$-action and finite automorphism constructed in Lemma 4.2. By Rentschler's theorem (see [Re]), there exists a system $(x, y)$ of polynomial coordinates and an element $P$ of $k[t]$ such that:

$$
\psi_{u}(x, y)=(x, y+u P(x))
$$

For any $f \in k[x, y]$, set $\operatorname{deg}_{\psi}(f)=\operatorname{deg}_{u} \exp u D(f)$. It is well-known that this defines a degree function on $k[x, y]$ (see [Da]). Since $\psi$ and $\Delta$ commute, $\Delta^{*}$ preserves the space $E_{n}$ of polynomials of degree $\leq n$ with respect to $\operatorname{deg}_{\psi}$. In particular, $\Delta^{*}$ preserves $E_{0}=k[x]$. So $\Delta^{*}$ induces a finite automorphism of $k[x]$, hence $\Delta^{*}(x)=a x+b$, where $a$ is a root of unity. Since $\Delta$ is finite, either $a \neq 1$ or $a=1$ and $b=0$. In any case, up to replacing $x$ by $x-\mu$ for a suitable constant $\mu$, we may assume that $\Delta^{*}(x)=a x$. Moreover $\Delta^{*}$ preserves the space $E_{1}=k[x]\{1, y\}$. With the same arguments as before, we obtain that $\Delta^{*}(y)=c y+d(x)$, where $c$ is a root of unity and $d(x)$ belongs to $k[x]$. Composing $\Delta$ with $\psi_{1 / m}$ then yields the second form given in Corollary 1.2 .

\section{Proof of Corollary 1.3}

Let $\Phi$ be an algebraic automorphism of $k^{2}$. We assume that $\Phi$ has a unique fixpoint $p$ and that $d \Phi_{p}$ is unipotent. We are going to prove that $n(\Phi)=0$.

First we check that $n(\Phi)$ cannot be equal to 2 . Assume that $n(\Phi)=2$. Then $k(x, y)^{\Phi}$ has transcendence degree 2 , and the extension $k(x, y) / k(x, y)^{\Phi}$ is algebraic, hence finite. Moreover $\Phi^{*}$ acts like an element of the Galois group of this extension. In particular, $\Phi^{*}$ is finite. By a result of Kambayashi (see [Ka]), $\Phi$ can be written as $h \circ A \circ h^{-1}$, where $A$ is an element of $G l_{2}(k)$ of finite order and $h$ belongs to $A u t\left(k^{2}\right)$. Since $\Phi$ has a unique fixpoint $p$, we have $h(0,0)=p$. In particular, $d \Phi_{p}$ is conjugate to $A$ in $G l_{2}(k)$. Since $d \Phi_{p}$ is unipotent and $A$ is finite, $A$ is the identity. Therefore $\Phi$ is also the identity, which contradicts the fact that it has a unique fixpoint.

Second we check that $n(\Phi)$ cannot be equal to 1 . Assume that $n(\Phi)=1$. By the previous corollary, up to conjugacy, we may assume that $\Phi$ has one of the following forms:

- $\Phi_{1}(x, y)=\left(a^{n} x, a^{m} b y\right)$, where $(n, m) \neq(0,0), b$ is a root of unity but $a$ is not,

- $\Phi_{2}(x, y)=(a x, b y+P(x))$, where $P$ belongs to $k[t]-\{0\}$ and $a, b$ are roots of unity.

Assume that $\Phi$ is an automorphism of type $\Phi_{1}$. Then $d \Phi_{p}$ is a diagonal matrix of $G l_{2}(k)$, distinct from the identity. But this is impossible since $d \Phi_{p}$ is unipotent. So assume that $\Phi$ is an automorphism of type $\Phi_{2}$. Then $d \Phi_{p}$ is a linear map of the form $(u, v) \mapsto(a u, b v+d u)$, with $d \in k$. Since $d \Phi_{p}$ is unipotent, we have $a=b=1$. So $(\alpha, \beta)$ is a fixpoint if and only 
if $P(\alpha)=0$. In particular, the set of fixpoints is either empty or a finite union of parallel lines. But this is impossible since there is only one fixpoint by assumption. Therefore $n(\Phi)=0$.

\section{An application of Corollary 1.3}

In this section, we are going to see how Corollary 1.3 can be applied to the determination of invariants for automorphisms of $\mathbb{C}^{3}$. Set $Q(x, y, z)=x^{2} y-z^{2}-x z^{3}$ and consider the following automorphism (see $[\mathrm{M}-\mathrm{P}])$ :

$$
\Phi: \mathbb{C}^{3} \longrightarrow \mathbb{C}^{3}, \quad\left(\begin{array}{l}
x \\
y \\
z
\end{array}\right) \longmapsto\left(\begin{array}{c}
x \\
y(1-x z)+\frac{Q^{2}}{4}+z^{4} \\
z-\frac{Q}{2} x
\end{array}\right)
$$

We are going to show that:

$$
\mathbb{C}(x, y, z)^{\Phi}=\mathbb{C}(x) \text { and } \quad \mathbb{C}[x, y, z]^{\Phi}=\mathbb{C}[x]
$$

Let $k$ be the algebraic closure of $\mathbb{C}(x)$. Since $\Phi^{*}(x)=x$, the morphism $\Phi^{*}$ induces an automorphism of $k[y, z]$, which we denote by $\Psi^{*}$. The automorphism $\Psi$ has clearly $(0,0)$ as a fixpoint, and its differential at this point is unipotent, distinct from the identity. Indeed, it is given by the matrix:

$$
d \Psi_{(0,0)}=\left(\begin{array}{cc}
1 & -x^{3} / 2 \\
0 & 1
\end{array}\right)
$$

Moreover, the set of fixpoints of $\Psi$ is reduced to the origin. Indeed, if $(\alpha, \beta)$ is a point of $k^{2}$ fixed by $\Psi$, then $x Q=0$ and $4 \beta^{4}-4 x \alpha \beta+Q^{2}=0$. Since $x$ belongs to $k^{*}$, we have:

$$
Q=x^{2} \alpha-\beta^{2}-x \beta^{3}=0 \text { and } \beta^{4}-x \alpha \beta=0
$$

If $\beta=0$, then $\alpha=0$ and we find the origin. If $\beta \neq 0$, then dividing by $\beta$ and multiplying by $-x$ yields the relation:

$$
x^{2} \alpha-x \beta^{3}=0
$$

This implies $\beta^{2}=0$ and $\beta=0$, hence a contradiction. By Corollary 1.3, the field of invariants of $\Psi$ has transcendence degree zero. So the field of invariants of $\Phi$ has transcendence degree $\leq 1$ over $\mathbb{C}$. Since this field contains $\mathbb{C}(x)$ and that $\mathbb{C}(x)$ is algebraically closed in $\mathbb{C}(x, y, z)$, we obtain that $\mathbb{C}(x, y, z)^{\Phi}=\mathbb{C}(x)$. As a consequence, the ring of invariants of $\Phi$ is equal to $\mathbb{C}[x]$.

\section{References}

[Bou] N.Bourbaki Eléments de Mathématiques: Algèbre Commutative, chapitres 5-6, Hermann Paris 1964. 
[Che] C.Chevalley Introduction to the theory of algebraic functions of one variable, Mathematical Surveys $n^{o}$ VI, American Mathematical Society, New York 1951.

[Da] D.Daigle On some properties of locally nilpotent derivations, J. Pure Appl. Algebra 114 (1997), $n^{0} 3,221-230$.

[Hum] J.Humphreys Linear algebraic groups, Graduate texts in mathematics 21, Springer Verlag Berlin 1981.

[Ka] T.Kambayashi Automorphism group of a polynomial ring and algebraic group action on an affine space, J.Algebra 60 (1979), no 2, 439-451.

[Kr] H.Kraft Challenging problems on affine n-space, Séminaire Bourbaki 802, 1994-95.

[M] L.Makar-Limanov On the hypersurface $x+x^{2} y+z^{2}+t^{3}=0$ in $\mathbb{C}^{4}$ or a $\mathbb{C}^{3}$-like threefold which is not $\mathbb{C}^{3}$, Israel J. Math. 96 (1996), part B, 419-429.

[M-P] L.Moser-Jauslin, P-M.Poloni Embeddings of a family of Danielewsky hypersurfaces and certain $(\mathbb{C},+)$-actions on $\mathbb{C}^{3}$, preprint.

[Re] R.Rentschler Opérations du groupe additif sur le plan affine, C.R.A.S 267 (1968) 384-387.

[Ro] M.Rosenlicht A remark on quotient spaces, An. Acad. Brasil. Cienc. 35 (1963), 487489.

[Ro2] M.Rosenlicht Automorphisms of function fields, Trans. Amer. Math. Soc. 79 (1955), $1-11$.

[Ru] P.Russell Forms of the affine line and its additive group, Pacific J. Math. 32 (1970) 527-539.

[S-B] G.Schwarz, M.Brion Théorie des invariants et Géométrie des variétés quotients, Collection Travaux en cours 61, Hermann Paris 2000.

[Van] A.Van den Essen Polynomial automorphisms and the Jacobian conjecture, Progress in Mathematics $n^{\circ} 190$, Birkhäuser Verlag, Basel 2000.

[V-P] A.Van den Essen, R. Peretz Polynomial automorphisms and invariants, J. Algebra 269 (2003), $n^{0} 1,317-328$.

[Z-S] O.Zariski, P.Samuel Commutative Algebra, Vol. II, The University Series in Higher Mathematics, D. Van Nostrand Co., Inc., Princeton, N.J.-Toronto-London-NewYork 1960. 